ANIMAL BEHAVIOUR

\section{Brain food}

Anyone studying for an exam knows that learning and memory cost energy. If energy is limited, cognitive functions can be impaired or traded off. Writing in Biology Letters, Jaumann et al. used honeybees (Apis mellifera) to assess whether changes in resource allocation at the group level might relieve such effects in group-living animals (S. Jaumann, R. Scudelari \& D. Naug Biol. Lett. http://dx.doi.org/10.1098/rsbl.2013.0149; 2013).

They find that learning imposes an energetic cost on individual honeybees from a colony, and that - just as in solitary animals — learning and retention suffer during starvation or following an immunological challenge.

A worldwide trend of colony collapse resulting from bee disappearance is worrying scientists. The authors suggest that diseases, pesticide exposure or habitat changes may impose energetic stresses, particularly on foraging bees, that impair their ability to find their way home. Marian Turner

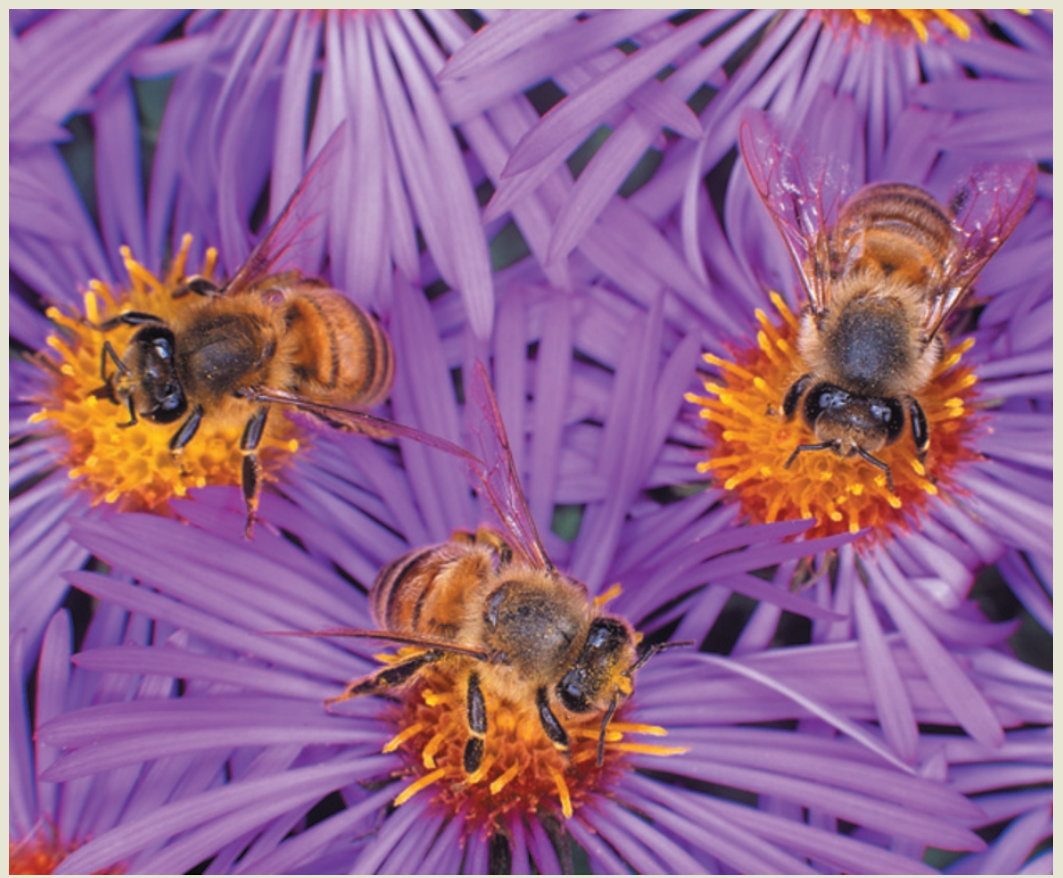

\title{
EPIDEMIOLOGY
}

\section{Resistance mapping in malaria}

\section{Whole-genome sequencing of human malaria parasites has revealed genomic regions that are associated with resistance to artemisinin-based drugs. The findings may help to explain the origin and spread of this worrying trend.}

\section{A. TAYLOR BRIGHT \& \\ ELIZABETH A. WINZELER}

A lthough malaria still causes more than one million deaths annually, the number of reported cases of the disease has been decreasing in recent years ${ }^{1,2}$. This decrease may be partly attributable to improved surveillance and control of mosquitoes, the malaria parasite's vector. But many experts feel that it is mainly a result of the availability and effectiveness of medicines called artemisinin-based combination therapies. Because this is the only class of antimalarial therapy that can effectively eliminate multi-drug-resistant malaria infections, the discovery of artemisinin resistance in parasites in western Cambodia ${ }^{3}$ has been viewed as a potential disaster by the international malaria community, and has sparked intensive effort to identify the genes conferring the resistance. Writing in Nature Genetics, Miotto et al. ${ }^{4}$ use whole-genome sequencing to investigate 825 samples of Plasmodium falciparum
- a common human malaria parasite - from southeast Asia and West Africa in an attempt to reveal the molecular basis and origin of artemisinin resistance.

There is currently no licensed malaria vaccine, so drugs remain the best way to control the infection. Artemisinin-based combination therapies (ACTs) are medicines in which one of the active ingredients is a derivative of artemisinin, a natural product originally isolated from the traditional Chinese medicinal herb sweet wormwood ${ }^{5}$. Although ACTs are very effective at treating malaria, their mechanism of action is not well understood, and resistance mechanisms seem to be different from those for other antimalarials. Resistance is also difficult to quantitate: no sensitive laboratorybased assays are available, and resistance is typically defined by how long it takes for malaria parasites to be cleared from a patient's body after treatment with artemisinin alone, a process in which patient immunity plays a part.

Furthermore, western Cambodia is the same region from which resistance to drugs based on chloroquine and sulfadoxine-pyrimethamine emerged and spread to the rest of the world in previous decades, leading to millions of extra deaths as these front-line antimalarials were rendered useless ${ }^{6-8}$. Fears of a repeat of this disaster if artemisinin-resistant parasites become widespread prompted the World Health Organization and other members of the Roll Back Malaria Partnership to launch the Global Plan for Artemisinin Resistance Containment (GPARC) in 2011 (go.nature. com/jgcpzc).

One of the main goals of GPARC is to identify the molecular basis of resistance to artemisinin, but this initiative has been confounded by the fact that this characteristic cannot be reliably assayed or reproduced in the laboratory. Instead, investigators have relied on genetic epidemiology - looking for signatures in parasite genomes that can shed light on the genetic basis of resistance. In these studies, parasite DNA is collected from infected people both in areas in which artemisinin resistance is common and in areas where it is not. By taking advantage of natural parasite sexual crosses that occur in the mosquito, together with conventional genotyping techniques or, most recently, whole-genome sequencing, genomic regions associated with resistant parasite populations can be mapped (Fig. 1). Genes present in these candidate regions are then prioritized for functional studies to validate what role, if any, they have in parasite resistance.

Using such techniques, Miotto et al. find evidence of three genetically distinct resistant parasite populations in a quite small geographic area, suggesting that resistance may have arisen independently at least three times. 